\title{
A statistical analysis of the soft X-ray profiles of solar flares
}

\section{G. Pearce ${ }^{1}$ and R.A. Harrison ${ }^{2}$}

1. Department of Astrophysics, Oxford University; Jesus College, Oxford; and Rutherford Appleton Lisbriatory

2. Astrophysics and Geoplýsics Division, Rutherford Appleton Laburatory

\section{listroduction}

We unclertale a statistical analysis of the solt $X$-ray $(3.5-5.5$ keV $)$ profiles of solar flares ats observed with the Ifard X-ray Inaging Spectroneter on the SMIM. The duratjons, maximun intensities and intensity" profiles of the flares are examined. The properties of the "typical" solat flare are discussed. The distrilutions of the neasured parameters with respect to one another reveal sonse interesting results. In common with past studies, we conclude that there is no evidence to suggest that more than one type of event is lueing viewed, despite a desire evident in the literature to place events into clistinct groups. We also conclude that commonly hedd views about the relatonships between flare duration and intensity, profile asjmunetries and intensity etc.. are in error. For more details of the flare events, the selection of data and the methud of analysis, the reader is referred to Pearce and Inarrison (1088).

For each of 59 flares, a $3.5-5.5 \mathrm{keV}$ time history plot was produced and varions paramulers tailien from it (as shown in Figure 1). We used the FIV(1/1)M value to musustue durations becallse it is contunonly sulticiently fiar above the backigrumed to be purely related to the flare. The parameters a and b give at uxeasure of the flare rise and clecay times, the duration $(a+b)$ and the asymetry of the prolile (b/a).

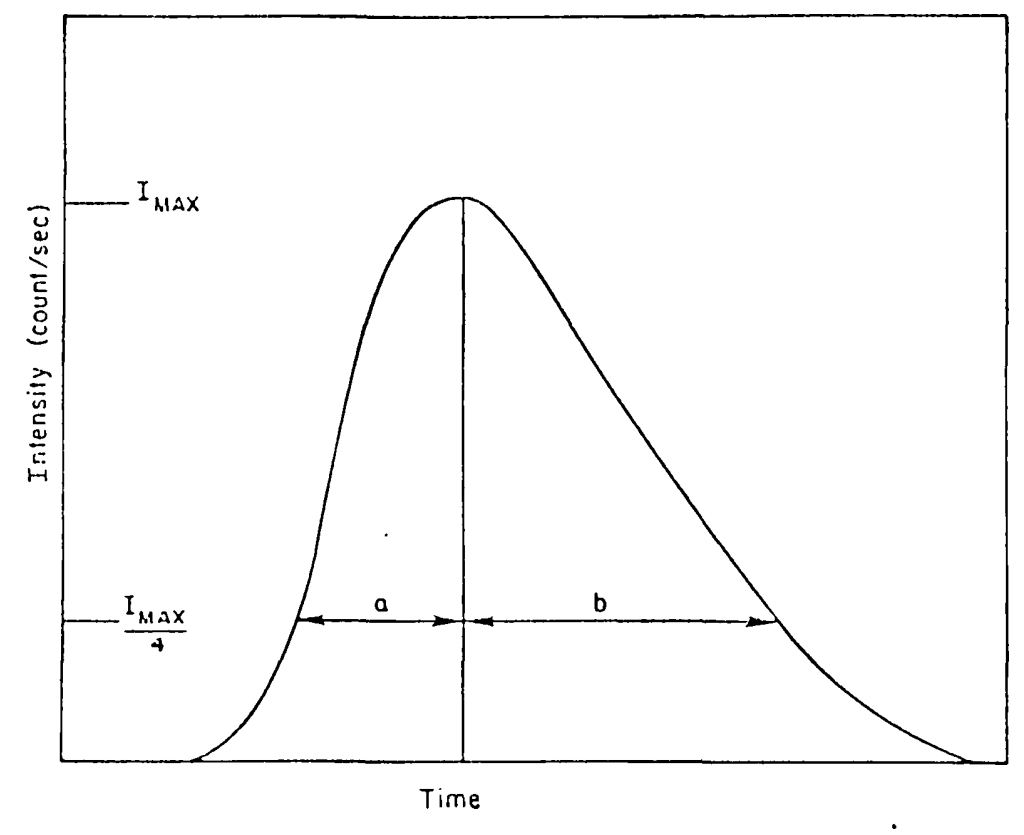

FIGURE 1: For cacle flare event the durations at and bo are meastured at the FIV $(1 / 4)$ M level of the 3.5-5.5 lia $V$ time-iutensity curve and the undximun intensity, $I_{\text {max }}$, i.s noted.

\section{Results}

Figure 2 shows a hislogram of number of flares versus flare duration (atb). It can be seen from this figure that the mode lies at $(a+b)$ between 5 and 10 ; the mean value is 1.1 .2 minutes. We mote that Phillips (1072) also found a mode between 5 and 10 minutes. Ifowever, in the present study we extend the analysis to longer clurations. Drake (1971) found a mode of 16 minutes.

Note that $72 \%$ of the X-ray events have durations betwen 5 and 20 minutes. The orerall shape of the plot resenubles a Puisson-lile curve with no sign of suls-grouping.s. This is consistent with Dralic (1071) and 
Phillips (1072). However, there is a selection effect due to the 60 minute orbital daylight period. If all flare durations were equally possible we should still expect a fall of: in Figure 2 simply because a longer event has a better cliance of truncation. However, the degree of this fall off can be estimated and has been corrected inset to Figure 2. However, the story does not change; there is still a maximum in the 5 to 20 minute region with a clear fall off and no sign of any distributions which could indicate the existence of another class of event. So, for the 1 to 60 minute duration region it secms that there is a continuous distribution.

The IIXIS had a minimum temporal resolution of about 3 seconds during most of the operations so we canuot look for micro-events of periods less than a few seconds. However, we believe that the fall off below 5 minutes is real. A similar fall-off at shorter durations was also identified by Drake (1071) and by Phillips (1972).

Figure 3 shows a histogram of number of flares versus flare asymmetry (b/a). There is a well defined peak at $b / a$ of about 1.5 . This indicates a surprising degrce of symmetry in the data-set. The mean value is a little more asymmetrical at 3.1. The histogram falls off smoothly up to the maximum of b/a about 10 . Note that we do see several events where the rise time is equal to or longer than the decay time $(b / a<1)$. Again, there is no indication of structure within the distribution. If there were two distinct classes such as long-lived asymmetrical events and short-lived symmetrical events we should see it reflected in this curve. The indications are that we see a continuous distribution due to one type of event.

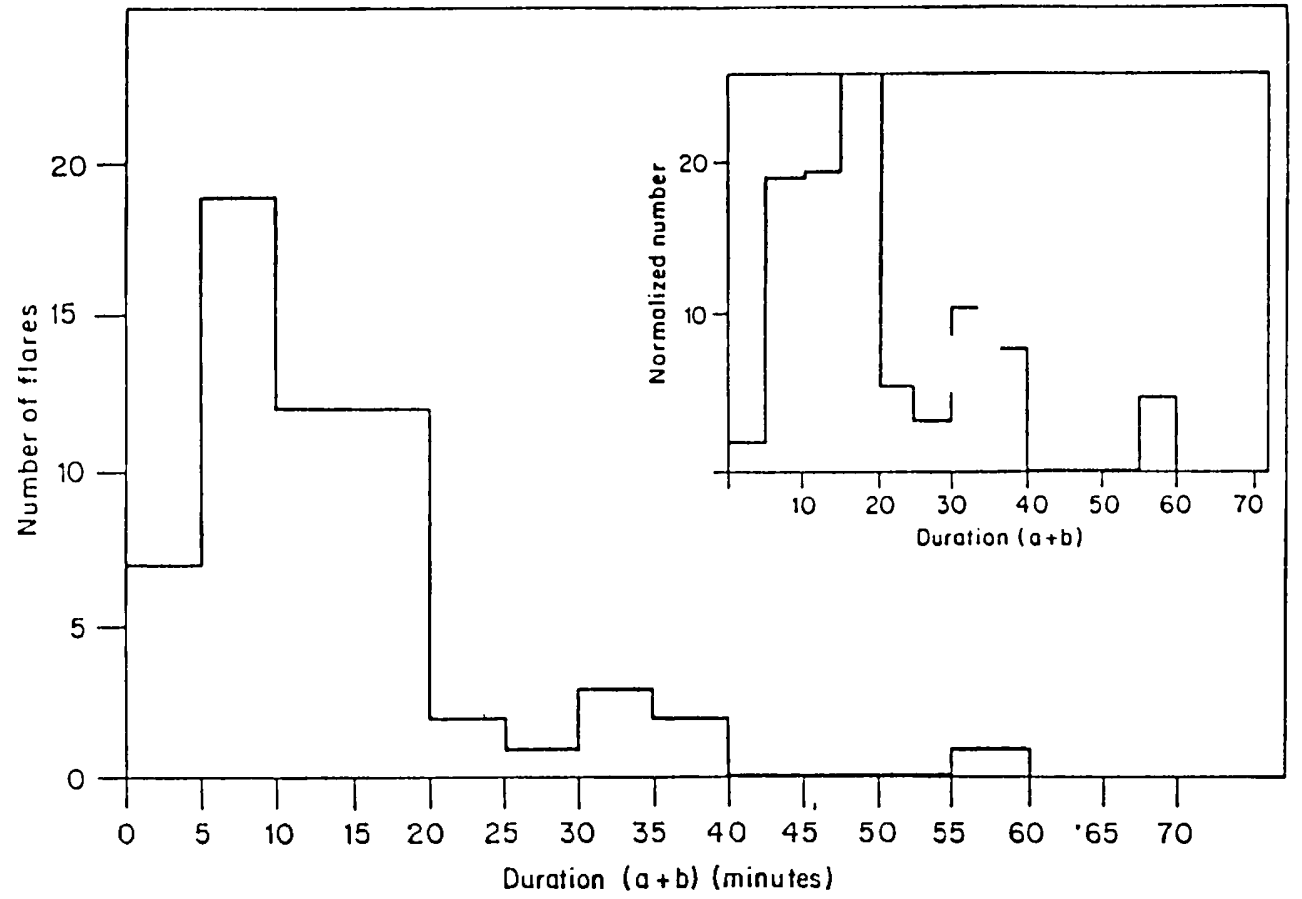

FIGURE 2: The distribution of flare durations $(a+b)$. The inset shows the same plot with the distribution normalized for effects of the orbital period.

Again, we can compare these data to those of Culhane and Phillips (1970) and Drake (1071). In both cases, a single-peaked distribution was found and this is supported by the HXIS data. In the former study the histogram of flare asymmetry peaked at (b/a) just above unity, which is consistent with our result. However, Drake (1971) found a greater asymmetry with a value nearer to 3.

Figure 4 slows a scatter-plot of the maximum flare intensity, $I_{\max }$, versus flare duration $(a+b)$. There is a wide scatter of points showing no applarent relationship. There is certainly no evidence for bunching into more than one class. The densely populated region at $(\mathrm{a}+\mathrm{b})=10$ to 20 (see Figure 2) in fact contains flares of all intensities. There is no evidence to state that brighter flares are longer-lived or weaker "ares short-lived. 
A similar plot of the variation of the maximum intensity of flares, $I_{\max }$, versus the degree of asymmetry of flares, $b / a$ also shows no particular relationship. Brighter flares are not necessarily more asymmetric and that weaker flares are not necessarily more symetrical.

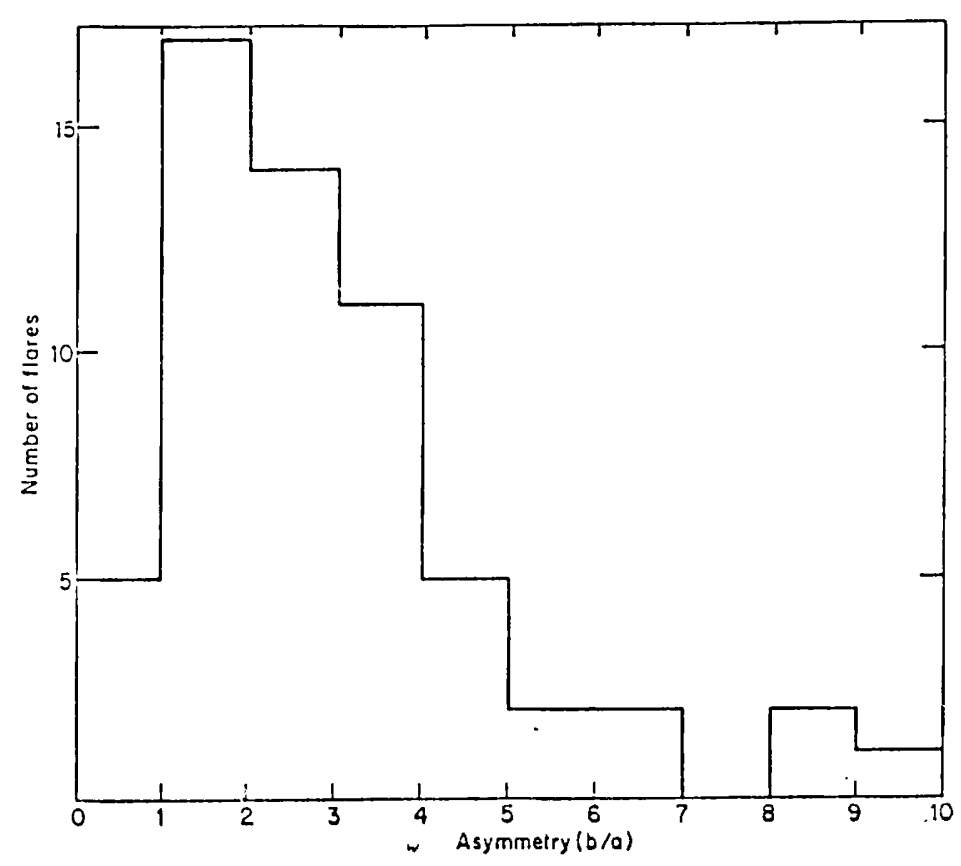

FIGURE 3: The distribution of the asymmetry of flare time-intensity profiles.

Figure 5 shows a plot of $b / a$ versus $(a+b)$. This illustrates the variation of flare asymmetry with flare duration. $67 \%$ of the flares plotted lie within the range $b / a<4$ and $a+b<20$. There is clearly a tendency to bunch in the lower left of the plot, though there is a good scatter. There are only 3 events with values of $\mathrm{b} / \mathrm{a}>7$. There is certainly no clear relationship between flare duration and asymmetry; longer lived flares are not necessarily more asymmetrical than short lived events and shorter lived events are not necessarily more symmetrical than longer lived events. The mean values for $b / a$ and $(a+b)$ are 3.1 and 14.2 minutes, respectively.

\section{Conclusions}

- There is no evidence for more than one distinct class of event as has been inferred by many reports in the literature. We find continuous distributions in intensity, duration and asymmetry.

The average flare FW(1/4)M duration was about 14 minutes with $72 \%$ of the events having durations $o i$ between 5 and 20 minutes. There is no evidence for a distinct long-duration event class in the distribution of durations and there was a clear fall off in the numbers of events at durations less than 5 minutes.

The average flare asymmetry, measured by the parameter b/a, is 3.1, though the mode is a surprisingly symmetrical 1.5. The distribution of asymmetries also showed no indication of class structure.

In plotting the flare maximum intensity versus the duration $(a+b)$ and the asymmetry $(b / a)$ we can state that there appeared to be no relationship in either case. It cannot be said that brighter flares are longer-lived or weaker flares short-lived. It cannot be said that brighter flares are more asymmetrical and weaker flares more symmetrical. These results contradict many commonly held beliefs.

There is no relationship between symmetry and duration. Longer-lived flares are not necessarily more asymmetrical and shorter-lived flares are not necessarily more symmetrical.

- One outcome of this analysis is the identification of the typical flare as seen in soft X-rays. The average values for $a$ and $b$, the nominal rise and decay times, were 4.1 and 10.0 minutes. The average value 
of $\mathrm{b} / \mathrm{a}$, the measure of asymmetry, was 3.1 , though the mode was an almost symmetrical 1.5 . The average FW(1/4)M duration was a little over 14 minutes.

FIGURE 4: A scatter plot showing maximum flare intensity versus flare duration $(a+b)$. The arrows indicate the directions in which minor motions of the data-points could occur in the cases where projections were made through periods of truncated data (see Table I).
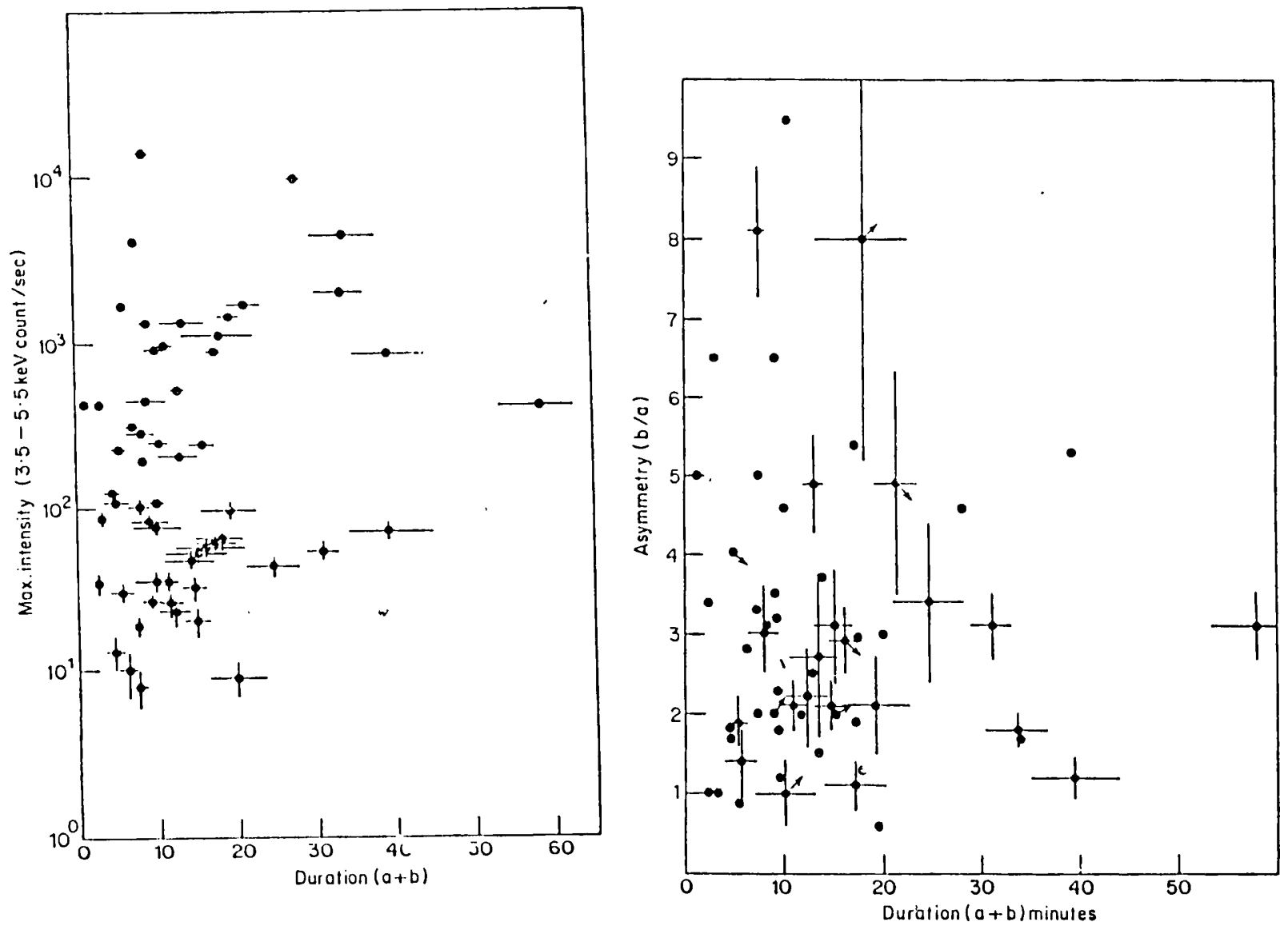
b).

FIGURE 5: A scatter plot showing the relationship between flare asymmetry (b/a) and duration $(\mathrm{a}+$

The results of this study rinforce those of studies produced during the last solar cycle. We find remarkable agrecment. This highlights the fallacy of attempting to subdivide flare activity. Finally, we conclude that a lesson has to be learnt from simple studies such as this. We need to examine events such as flares in perspective, which means keeping a "statistical" eye on our prejudices. Despite similar studies in the previous solar eycle, our analyses of the last solar maximum indicate a disregard for the overall picture.

\section{Acknowledgements}

GP would like to express thanks to Jesus College, Oxford University and the Rutherford Appleton Laboratory for an Atlas Research Fellowship.

\section{References}

Culhane, J.L. and Phillips, K.J.H.; 1970, Solar Phys. 11, 117.

Drake, J.F.: 1971, Solar I'!ys. 16, 152.

Pearce, G. and Harrison, R.A.: 1988, Astron. Astrophys. in press.

Phillips, K.J.H.: 1972, Ph.D. Thesis, University College London. 